\title{
First-principles calculations for nitrogen-containing single-walled carbon nanotubes
}

James P. Lewis

Mingwen Zhao

Yueyugan Xia

Ruiqin Zhang

Follow this and additional works at: https://scholarsarchive.byu.edu/facpub

Part of the Astrophysics and Astronomy Commons, and the Physics Commons

\section{Original Publication Citation}

Zhao, Mingwen, Yueyuan Xia, James P. Lewis, and Ruiqin Zhang."First-principles calculations for nitrogen-containing single-walled carbon nanotubes." Journal of Applied Physics 94 (23):

2398-242.

\section{BYU ScholarsArchive Citation}

Lewis, James P.; Zhao, Mingwen; Xia, Yueyugan; and Zhang, Ruiqin, "First-principles calculations for nitrogen-containing single-walled carbon nanotubes" (2003). Faculty Publications. 482.

https://scholarsarchive.byu.edu/facpub/482 


\title{
First-principles calculations for nitrogen-containing single-walled carbon nanotubes
}

\author{
Mingwen Zhao $^{\mathrm{a})}$ and Yueyuan Xia \\ Department of Physics, Shandong University, Jinan 250100, China
}

James P. Lewis

Department of Physics and Astronomy, Brigham Young University, Provo, Utah 84602-4658

Ruiqin Zhang

Center of Super-Diamond and Advanced Films (COSDAF) and Department of Physics and Materials Science, City University of Hong Kong SRA, China

(Received 15 November 2002; accepted 2 June 2003)

\begin{abstract}
We present calculations for possible configurations of nitrogen-containing single-walled carbon nanotubes and their electronic properties obtained with the $a b$ initio tight-binding FIREBALL method. It is found that nitrogen atoms can be energetically incorporated into the carbon network in three forms: Substitution, substitution with formation of a vacancy structure, and chemical adsorption. The different forms exhibit different local densities of states near the Fermi levels, which might suggest a potential method to control the electronic properties of nitrogen-doped carbon nanotubes. (C) 2003 American Institute of Physics. [DOI: 10.1063/1.1593798]
\end{abstract}

\section{INTRODUCTION}

There has been much interest in the mechanical, electronic, and structural properties of carbon nanotubes since their discovery. ${ }^{1}$ Their unique properties make them a very promising candidate for building blocks of molecular-scale machines and nanoelectronic devices. ${ }^{2-4}$ Single-walled carbon nanotubes (SWNTs) can be either metallic or semiconducting, depending on the chiral vectors $(n, m){ }^{5,6}$ Furthermore, the introduction of topological defects, such as pentagon-heptagon pairs, into the hexagonal network of a SWNT can change the helicity of the tube, altering its electronic structure. It is proposed that nanoscale heterojunctions can be obtained within a single SWNT through this method. ${ }^{7,8}$

Adsorption of many kinds of atoms or molecules, such as $\mathrm{F}, \mathrm{K}, \mathrm{O}_{2}, \mathrm{H}_{2}$, and $\mathrm{NO}_{2}$, on carbon nanotubes also changes their electronic properties. ${ }^{9-13}$ This suggests a potential application of carbon nanotubes as a chemical sensor or more generally a method for controlling the electronic properties of carbon nanotubes by modifying their structure through doping with different atoms.

With the development of synthesizing techniques, doped carbon nanotubes have attracted more and more attention. Others have shown that doped SWNTs exhibit conductivity enhancement and charge transfer. ${ }^{14,15}$ In addition, significant enhancement of the local density of states (LDOS) at the Fermi level caused by introducing substitutional boron or nitrogen atoms into the tube tip of SWNTs was predicted by using first-principles calculations. ${ }^{16}$ Nitrogen is an ideal doping atom, for it can be easily incorporated into the carbon

\footnotetext{
a) Author to whom correspondence should be addressed; also at: Center of Super-Diamond and Advanced Films (COSDAF) and Department of Physics and Materials Science, City University of Hong Kong SRA, China; electronic mail: zmw@sdu.edu.cn
}

network through many different techniques, such as substitution reactions, ${ }^{17,18}$ electric-arc evaporation, and magnetron sputtering. ${ }^{19-21}$ The nitrogen content ranges from 5\% to $30 \%$ in nitrogen-containing carbon nanotubes prepared by different techniques. ${ }^{19,20}$ The electronic structure of nitrogencontaining carbon nanotubes has been determined from the analysis of the $\mathrm{x}$-ray photoelectron and $\mathrm{x}$-ray fluorescence spectra. ${ }^{21}$ From the $\mathrm{N} 1 s$ photoelectron spectrum of nitrogencontaining carbon nanotubes, it is proposed that nitrogen atoms can be incorporated into the carbon network with the formation of a vacancy defect, where each doping nitrogen atom is bonded to two carbon atoms. Other nitrogencontaining carbon nanotubes are energetically promising, especially in consideration of predicted results on azafullerene structures containing $20 \%$ nitrogen $\left(\mathrm{C}_{48} \mathrm{~N}_{12}\right) .{ }^{22}$

There have been few theoretical efforts to study the nanostructure and electronic properties of nitrogencontaining carbon nanotubes. In this article, we investigated the possible configurations of nitrogen-containing carbon nanotubes and their LDOS by using electronic structure calculations. The results show that nitrogen atoms can be stably incorporated into carbon nanotubes in three different forms, and exhibit different electronic properties.

\section{METHODS}

We use the $a b$ intitio tight-binding program FIREBALL, ${ }^{23}$ based on the density-functional theory (DFT) and a nonlocal pseudopotential scheme, for our atomistic simulations of nitrogen-containing SWNTs. The basis functions used are a set of slightly excited pseudoatomic fireball wave functions. These orbitals are computed within the DFT and the generalized norm-conserving separable pseudopotentials of the Hamann form are used ${ }^{24}$ employing, for their construction, the scheme of Fuchs and Scheffler. ${ }^{25}$ The FIREBALl wave functions are chosen such that they vanish at some radius $r_{c}$. 
We choose a double numerical (DN) basis set $s s^{*} p p^{*}$ for nitrogen and a single numerical basis set $s p^{3}$ for carbon. These basis sets have been shown to provide reasonable accuracy with a low demand on computation resources. The form of the DN basis sets, that we use, are prescribed by Delley where the wave functions of the $2^{+}$ions determine the excited state orbitals. ${ }^{26}$ The localized pesudoatomic orbitals were constructed with a confinement radius of $3.70 a_{B}$ for the $2 s$ state and $4.10 a_{B}$ for the $2 p$ state of nitrogen, while the $2 s$ and $2 p$ orbitals of carbon were confined in a sphere with radius of $4.00 a_{B}$ and $4.40 a_{B}$, respectively.

The model of electronic exchange-correlation functional employed in these calculations was the generalized gradient approximation (GGA). We use the GGA form containing Becke exchange (B88) with Lee-Yang-Parr (LYP) correlation. ${ }^{27,28}$ Periodical boundary conditions along the tube axis were used for SWNTs. The supercell selected for the $(5,5)$ SWNT contains ten layers of carbon atoms $(100$ atoms). All of the equilibrium configurations presented herein were obtained by full relaxation until the energy convergence reached a tolerance of $1.0 \times 10^{-3} \mathrm{eV} / \mathrm{atom}$. The cohesive energy $E_{c}$ of a pure SWNT and nitrogen-containing SWNT is calculated from its total energy $E_{T}$ and the energy of a single free carbon atom $\left(E_{1}\right)$ and nitrogen atom $\left(E_{2}\right)$ using the following equation: $E_{c}=E_{T}-n_{1} E_{1}-n_{2} E_{2}$, where $n_{1}$ and $n_{2}$ are the number of carbon atoms and nitrogen atoms, respectively.

\section{RESULTS AND DISCUSSION}

Nitrogen can be incorporated into the hexagonal network of SWNTs through the substitution of nitrogen for carbon. The five valence electrons of nitrogen can form three $\mathrm{C}-\mathrm{N}$ bonds with the neighboring carbon atoms; however, there are differences in bond length and binding energy between the $\mathrm{C}-\mathrm{N}$ bond and $\mathrm{C}-\mathrm{C}$ bond. Calculations at the B3 LYP/6-31G* level within GAUSSIAN98 ${ }^{29}$ yield equilibrium bond lengths of $\mathrm{C}-\mathrm{N}$ and $\mathrm{C}_{2}$ dimers at $1.17 \AA$ and $1.25 \AA$, respectively, while the difference in the binding energy between a $\mathrm{C}-\mathrm{N}$ and $\mathrm{C}_{2}$ dimer is $-2.48 \mathrm{eV}$. Therefore, the incorporation of nitrogen atoms into the hexagon network of SWNTs will cause structural distortion of the tube wall around the substitutional sites, but such a substitution is more energetically favorable.

Our calculated equilibrium configuration of a $(5,5)$ SWNT with a single substitutional nitrogen atom [shown in Fig. 1(a)], shows similar trends as in the dimer case. The average $\mathrm{C}-\mathrm{N}$ bond length in the nitrogen-substituted $(5,5)$ SWNT is $1.42 \AA$, which is shorter than the averaged bond length, $1.46 \AA$, of $\mathrm{C}-\mathrm{C}$ bonds of the SWNT. The difference of the cohesive energy between a $(5,5)$ SWNT with a substitutional nitrogen atom and a pure $(5,5) \mathrm{SWNT}$ is $-2.23 \mathrm{eV}$.

We now consider the cohesive energies in different configurations of nitrogen-substituted $(5,5)$ SWNTs with more than one substitutional nitrogen atom, as shown in Figs. 1(b)-1(f). These cases correspond to two adjacent nitrogen atoms [Fig. 1(b)], two nonadjacent nitrogen atoms placed on different sites of the hexagon [Fig. 1(c)-1(e)], and three nonadjacent nitrogen atoms [Fig. 1(f)]. The cohesive energies of

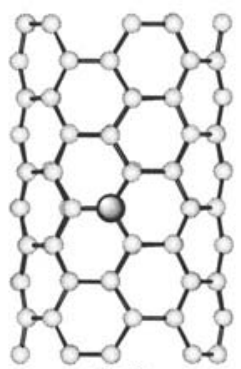

(a)

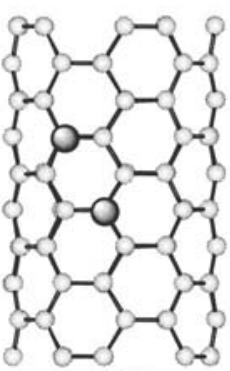

(d)

\section{○ Nitrogen \\ - Carbon}

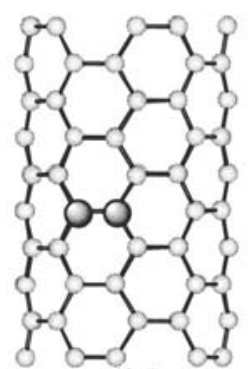

(b)

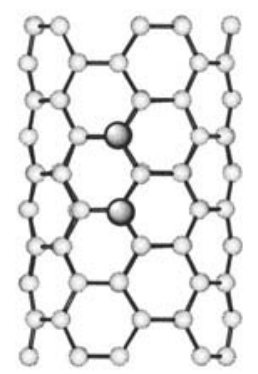

(e)

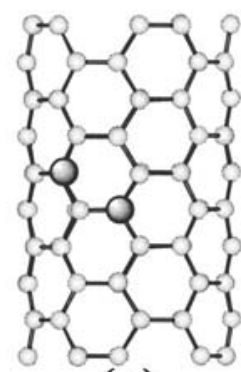

(c)

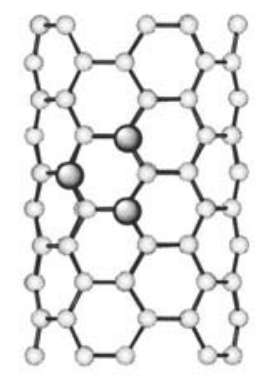

(f)
FIG. 1. The possible configurations of nitrogen-containing $(5,5)$ SWNT.

these nitrogen-substituted $(5,5)$ SWNTs (difference from pure carbon nanotube) are $2.02 \mathrm{eV},-3.86 \mathrm{eV},-3.73 \mathrm{eV}$, $-3.09 \mathrm{eV}$, and $-5.21 \mathrm{eV}$, respectively. It is clear that the configuration containing two adjacent substitutional nitrogen atoms, as shown in Fig. 1(b), is energetically unfavorable, and therefore unlikely appears in nitrogen-containing SWNTs. The cohesive energy of nitrogen-substituted $(5,5)$ SWNTs decreases with the increase in the number of substitutional nitrogen atoms. This does not necessarily mean that the nitrogen-substituted SWNT with high nitrogen content is more probable because the nitrogen content in SWNTs will depend on many factors, such as synthesis techniques. ${ }^{21}$

We next construct the configurations of $(5,5)$ SWNT with $20 \%$ and $40 \%$ substitutional nitrogen atoms, where the distribution of nitrogen is uniformly placed such that no two nitrogen atoms are adjacent. The calculated equilibrium configurations of the two tubes are represented in Figs. 2(a) and 2 (b), respectively. The distortion of the $(5,5)$ SWNT containing $20 \%$ nitrogen atoms is slight; however, remarkable distortion occurs as the nitrogen content is up to $40 \%$. In both of these cases, the axis of the tubes remain straight during optimization processes, although there is obvious radial distortion.

Additionally, we simulated the annealing of a $(5,5)$ SWNT containing 18 substitutional nitrogen atoms (200 atoms total), which were distributed in a local area on the tube wall. After being annealed at $500 \mathrm{~K}$ for $10 \mathrm{ps}$, the tube bent substantially, as shown in Fig. 2(c). It can be expected that with the increase of substitutional atoms, longer carbon nanotubes may roll up to form a more crooked structure. 


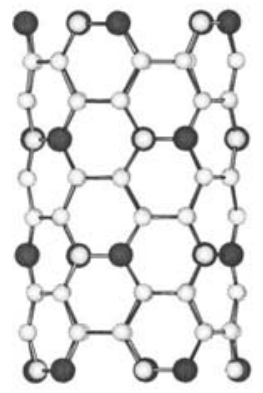

(a)

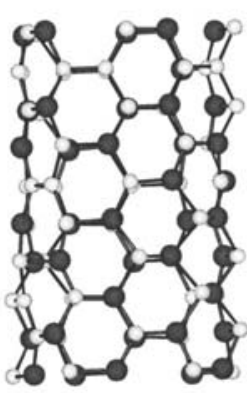

(b)
Nitrogen

Carbon

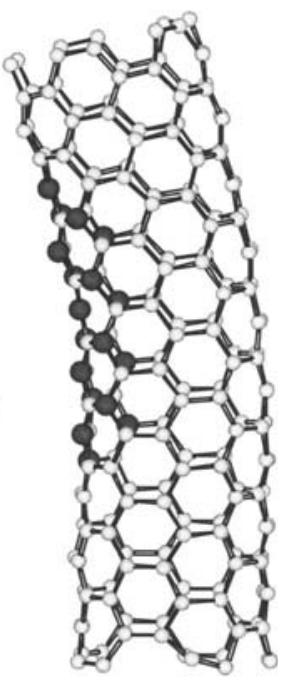

(c)
FIG. 2. The configurations of $(5,5)$ SWNT containing (a) $20 \%$, (b) $40 \%$, and (c) $9 \%$ nitrogen atoms. The substitutional atoms are uniformly distributed in (a) and (b).

This result is consistent with the transmission electron microscopy (TEM) images of nitrogen-containing carbon nanotubes where the graphitic network of a nitrogen-rich carbon nanotube is strongly distorted and partially stratified. ${ }^{21}$

We next consider the configuration of a nitrogen atom incorporated into a $(5,5)$ SWNT with the addition of an adjacent vacancy, as shown in Fig. 3(a). Carbon nanotubes usually contain a considerable amount of defects, such as vacancies in the network of carbon hexagons. ${ }^{30,31}$ It has been assumed that the nitrogen atoms can be incorporated into the carbon network with the formation of a vacancy structure. ${ }^{21}$ But, no other detailed knowledge of such vacancy structures has been presented. In our calculated vacancy structure, the substitutional nitrogen is incorporated into a hexagon, and bonded to two carbon atoms, creating a carbon vacancy; the optimized structure is shown in Fig. 3(a). We find the bond length of the $\mathrm{C}-\mathrm{N}$ bond to be $1.34 \AA$, which is much shorter than the substitutional $\mathrm{C}-\mathrm{N}$ bond length and more like the

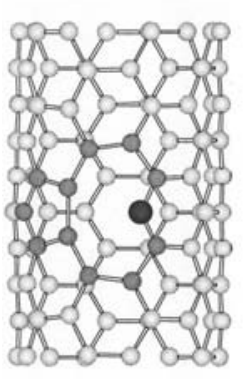

(a)

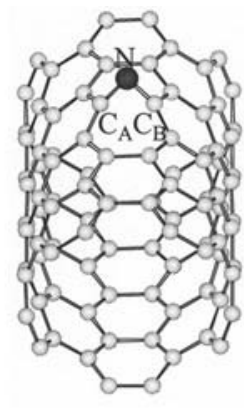

(b)

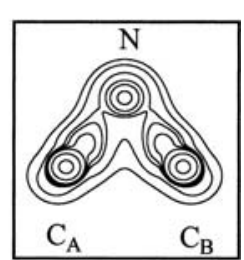

Nitrogen

- Carbon
FIG. 3. (a) The configuration of $(5,5)$ SWNT with a substitutional nitrogen which is incorporated into the carbon network with formation of a vacancy structure. (b) The geometric structure of $(5,5)$ SWNT with an adsorbed nitrogen atom.

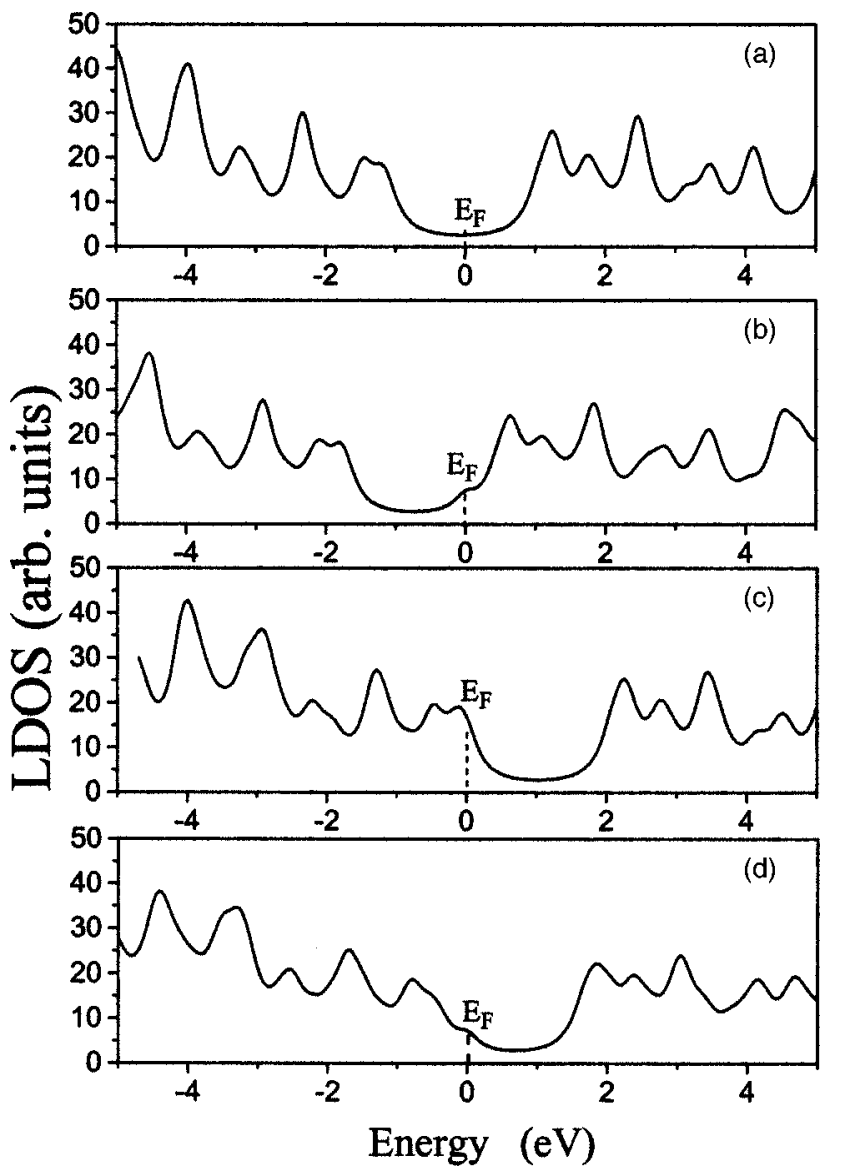

FIG. 4. The LDOS near the Fermi level of different nitrogen-doped SWNT configurations. The horizontal axis represents energy, where the energy at Fermi level is set as zero: (a) pure $(5,5)$ SWNT, (b) $(5,5)$ SWNT with a substitutional nitrogen atom, (c) $(5,5)$ SWNT with an adsorbed nitrogen atom, and (d) $(5,5)$ SWNT containing a substitutional nitrogen which is incorporated into the carbon network with formation of a vacancy structure.

length of a double bond. The electronic state of the nitrogen atom with this vacancy is definitively different from that of the substitutional nitrogen atom structure where each nitrogen atom is bonded to three carbon atoms.

Previous studies have demonstrated that a single nitrogen atom can be chemically adsorbed on the exterior surface of small radius SWNTs, and the most possible adsorption site is over a $\mathrm{C}-\mathrm{C}$ bond. ${ }^{32}$ The equilibrium structure of a $(5,5)$ SWNT with an adsorbed nitrogen atom is shown in Fig. 3(b). From Fig. 3(b), it is evident that there is remarkable distortion of the wall of the SWNT around the adsorption site. The distance between the two carbon atoms $\mathrm{C}_{\mathrm{A}}$ and $\mathrm{C}_{\mathrm{B}}$ (bonded to the adsorbed nitrogen) is elongated to $1.81 \AA$ from a bond length of $1.46 \AA$. The electronic density contours on a plane containing the adsorbed nitrogen and its neighboring carbon atoms are depicted in the inset of Fig. 3(b). From the inset of Fig. 3(b), it is obvious that the covalent bond between the carbon atoms, $\mathrm{C}_{\mathrm{A}}$ and $\mathrm{C}_{\mathrm{B}}$, is broken, as each carbon atom now forms $\mathrm{C}-\mathrm{N}$ covalent bonds. The adsorption energy of a nitrogen atom on the exterior wall of $(5,5)$ SWNT is calculated to be $-4.31 \mathrm{eV}$; thus, the nitrogen adsorption is a chemical adsorption and therefore is stable.

We calculate the LDOS near the Fermi levels from some of our different configurations and present the results in Fig. 


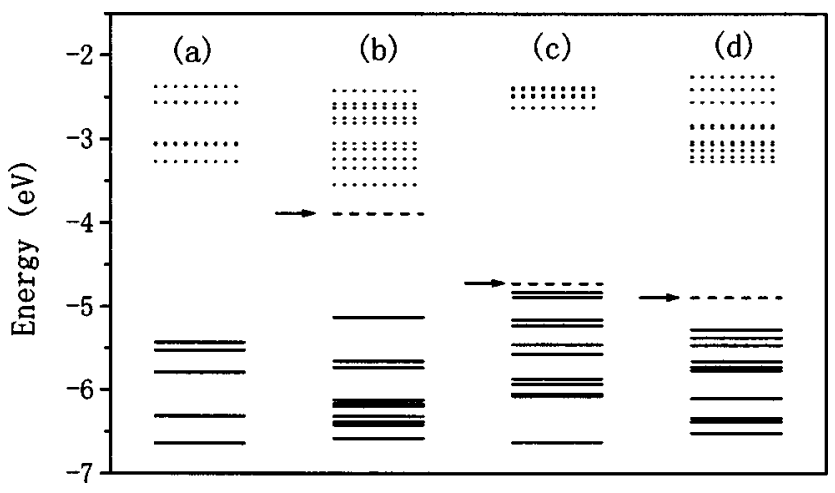

FIG. 5. Energy levels around the Fermi level for (a) pure $(5,5)$ SWNT, (b) $(5,5)$ SWNT with a substitutional nitrogen atom, (c) $(5,5)$ SWNT with an adsorbed nitrogen atom, and (d) (5,5) SWNT containing a substitutional nitrogen which is incorporated into the carbon network with formation of a vacancy structure. Solid lines and dot lines correspond to fully occupied levels and unoccupied levels, respectively. The dashed lines represent the half-occupied levels.

4. The LDOS of a pure $(5,5)$ SWNT is depicted in Fig. 4(a) for reference. With a substitutional nitrogen atom [configuration in Fig. 1(a)], the Fermi level is shifted higher in the LDOS, as shown in Fig. 4(b), such that the Fermi level of the substitutional nitrogen atom configuration is above the Fermi level of the pure $(5,5)$ SWNT. This indicates that $(5,5)$ SWNT with a substitutional nitrogen atom would be regarded as a $n$-type-substituted carbon nanotube. This result is related to the nature of the substitutional nitrogen atom, which has five valence electrons. Noting that three carbon atoms are bonded to the nitrogen, the substitutional nitrogen atom, therefore, can be regarded as a donor. Contrary to the previous case, the $(5,5)$ SWNT configuration with an adsorbed nitrogen atom [configuration in Fig. 3(b)], has its Fermi level shifted lower, as shown in Fig. 4(c), compared to the pure $(5,5)$ SWNT. This indicates that the $(5,5)$ SWNT with an adsorbed nitrogen atom would be regarded as a $p$-type-adsorbed carbon nanotube. This result can be explained by the fact that only two carbon atoms are bonded to the nitrogen atom, and the nitrogen can be regarded as an acceptor. For the LDOS of a nitrogen-substituted $(5,5)$ SWNT with a vacancy structure [configuration in Fig. 3(a)], shown in Fig. 4(d), this kind of nitrogen-substituted tube is also a $p$-type-substituted carbon nanotube.

The energy levels around the Fermi level for the pure $(5,5)$ SWNT and nitrogen-containing $(5,5)$ SWNTs are shown in Fig. 5. Half-occupied levels, represented by the dashed lines in Fig. 5, appear in the energy gap of the nitrogen-containing SWNTs. These levels are related to the way nitrogen is incorporated into the SWNTs. For the nitrogen-substituted $(5,5)$ SWNT, the half-occupied level can be regarded as a donor level since it is close to the unoccupied levels. However, for the SWNTs where a nitrogen atom is incorporated through chemical adsorption or substitution with formation of vacancy, this half-occupied level is near the fully occupied levels, and thus acts as an acceptor level.

From the analysis given herein, we deduce that nitrogen incorporated into carbon nanotubes with formation of vacancies, or for nitrogen chemically adsorbed, the nitrogen-

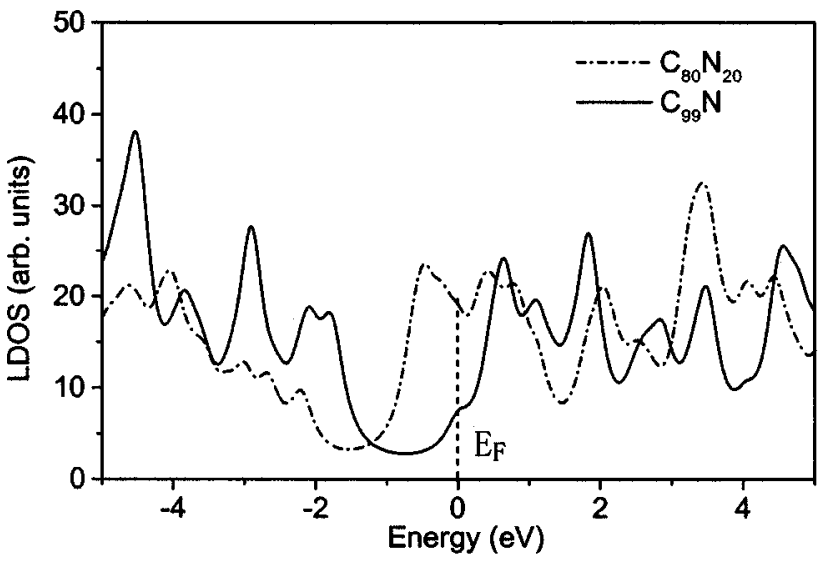

FIG. 6. The LDOS of $(5,5) \mathrm{SWNT}$ with a substitutional nitrogen atom (solid line), and (5,5) SWNT containing 20\% substitutional nitrogen atoms (dashed line)

containing carbon nanotubes will act as $p$-type-doped carbon nanotubes. Conversely, the other considered nitrogencontaining SWNTs can be regarded as $n$-type-substituted carbon nanotubes provided that most of nitrogen atoms are incorporated into the SWNT through substitution without vacancy. This suggests a method to control the electronic property of SWNTs by adjusting nitrogen doping methods. More importantly, if the three different forms of nitrogen atoms are doped into different regions of a single SWNT, it is possible to form heterojunctions within the carbon nanotube.

The LDOS near their Fermi levels of a $(5,5)$ SWNT with a substitutional nitrogen atom $\left(\mathrm{C}_{99} \mathrm{~N}\right)$ and a $(5,5)$ SWNT with $20 \%$ substitutional nitrogen atoms $\left(\mathrm{C}_{80} \mathrm{~N}_{20}\right)$ are shown in Fig. 6. It is obvious that the LDOS of $\mathrm{C}_{80} \mathrm{~N}_{20}$ at the Fermi level is greatly enhanced compared with that of $\mathrm{C}_{99} \mathrm{~N}$. As it is well known, the LDOS near the Fermi level directly affects the chemical reactivity and electronic properties, such as field emission and electronic conductivity. ${ }^{16}$ Therefore, these LDOS show that increasing nitrogen content into SWNTs will cause higher chemical reactivity and electronic conductivity.

\section{CONCLUSIONS}

In summary, nitrogen atoms can be incorporated into carbon nanotubes in three forms: Substitution, substitution with formation of vacancy structure, and chemical adsorption. The incorporation of nitrogen atoms can induce structural distortions of carbon nanotubes, which ultimately may cause the carbon nanotubes to roll up to form a more crooked structure. Nitrogen-substituted $(5,5)$ SWNT without a vacancy defect can be regarded as a $n$-type-substituted carbon nanotubes. However, if nitrogen atoms are incorporated into a carbon network with the formation of a vacancy included, the nitrogen-containing $(5,5)$ SWNT acts as a p-typesubstituted carbon nanotube. The $(5,5)$ SWNT with an adsorbed nitrogen atom is also a $p$-type nanotube. In general, the chemical reactivity and electronic conductivity of nitrogen-containing carbon nanotubes increase with the nitrogen content. 


\section{ACKNOWLEDGMENTS}

This work is supported by the National Natural Science Foundation of China under Grant Nos. 10175038 and 90203013, and the Foundation of Ministry of Education of China under grants No. 20020422012.

${ }^{1}$ S. Iijima, Nature (London) 354, 56 (1991).

${ }^{2}$ M. M. J. Treacy, T. W. Ebbsen, and J. M. Gibson, Nature (London) 381, 678 (1996)

${ }^{3}$ M. R. Falvo, G. J. Clary, R. M. Taylor, V. Chi, F. P. Brooks, S. Washburn, and R. Superfine, Nature (London) 389, 582 (1997).

${ }^{4}$ E. W. Wong, P. E. Sheehan, and C. M. Lieber, Science 277, 1971 (1997).

${ }^{5}$ R. Saito, M. Fujita, G. Dresselhaus, and M. S. Dresselhaus, Appl. Phys. Lett. 60, 2204 (1992).

${ }^{6}$ N. Hamada, S. Sawada, and A. Oshiyama, Phys. Rev. Lett. 68, 1579 (1992).

${ }^{7}$ L. Chico and V. H. Crespi, Phys. Rev. Lett. 76, 971 (1996).

${ }^{8}$ T. W. Ebbesen and T. Takada, Carbon 33, 973 (1995).

${ }^{9}$ J. Kong and N. R. Franklin, Science 287, 622 (2000).

${ }^{10}$ C. Jo, C. Kim, and Y. H. Lee, Phys. Rev. B 65, 035420 (2000).

${ }^{11}$ N. Park, S. Han, and J. Ihm, Phys. Rev. B 64, 125401 (2001).

${ }^{12}$ A. Maiti, J. Andzelm, N. Tanpipat, and P. von Allmen, Phys. Rev. Lett. 87, 155502 (2001)

${ }^{13}$ O. Gulseren, T. Yildirim, and S. Ciraci, Phys. Rev. Lett. 87, 116802 (2001).

${ }^{14}$ R. S. Lee, H. J. Kim, J. E. Fischer, A. Thess, and R. E. Smalley, Nature (London) 388, 255 (1997).

${ }^{15}$ A. M. Rao, P. C. Eklund, S. Bandow, A. Thess, and R. E. Smalley, Nature (London) 388, 257 (1997).

${ }^{16}$ G. Zhang, W. H. Duan, and B. L. Gu, Appl. Phys. Lett. 80, 2589 (2002).

${ }^{17}$ D. Golberg, Y. Bando, W. Han, K. Kurashima, and T. Sato, Chem. Phys. Lett. 308, 337 (1999).

${ }^{18}$ W. Han, Y. Bando, K. Kurashima, and T. Sato, Chem. Phys. Lett. 299, 368 (1999).
${ }^{19}$ O. Stephan, P. M. Ajayan, C. Collex, P. Redlich, J. M. Lambert, P. Bernier, and P. Lefin, Science 266, 1683 (1994).

${ }^{20}$ K. Suenaga, M. P. Johansson, N. Hellgren, E. Broitman, L. R. Wallenberg, C. Colliex, J. E. Sundgren, and L. Hultman, Chem. Phys. Lett. 300, 695 (1999).

${ }^{21}$ A. G. Kudashov, A. V. Okotrub, N. F. Yudanov, A. I. Romanenko, L. G. Bulusheva, O. G. Abrosimov, A. L. Chunvilin, E. M. Pazhetov, and A. I. Boronin, Phys. Solid State 44, 652 (2002).

${ }^{22}$ R. Manaa, J. Am. Chem. Soc. 124, 13990 (2002).

${ }^{23}$ J. P. Lewis, K. R. Glaesemann, G. A. Voth, J. Fritsch, A. A. Demkov, J. Ortega, and O. F. Sankey, Phys. Rev. B 64, 195103 (2001).

${ }^{24}$ D. R. Hamann, Phys. Rev. B 40, 2980 (1989).

${ }^{25}$ M. Fuchs and M. Scheffler, Comput. Phys. Commun. 119, 67 (1999).

${ }^{26}$ B. Delley, J. Chem. Phys. 92, 508 (1990).

${ }^{27}$ A. D. Becke, Phys. Rev. A 38, 3098 (1988).

${ }^{28}$ C. Lee, W. Yang, and R. G. Parr, Phys. Rev. B 37, 785 (1988).

${ }^{29}$ M. J. Frisch, G. W. Trucks, H. B. Schlegeli, E. Scuseria, M. A. Robb, J. R. Cheeseman, V. G. Zakrzewski, J. A. Montgomery, Jr., R. E. Stratmann, J. C. Burant, S. Dapprich, J. M. Millam, A. D. Daniels, K. N. Kudin, M. C. Strain, O. Farkas, J. Tomasi, V. Barone, M. Cossi, R. Cammi, B. Mennucci, C. Pomelli, C. Adamo, S. Clifford, J. Ochterski, G. A. Petersson, P. Y. Ayala, Q. Cui, K. Morokuma, D. K. Malick, A. D. Rabuck, K. Raghavachari, J. B. Foresman, J. Cioslowski, J. V. Ortiz, B. B. Stefanov, G. Liu, A. Liashenko, P. Piskorz, I. Komaromi, R. Gomperts, R. L. Martin, D. J. Fox, T. Keith, M. A. Al-Laham, C. Y. Peng, A. Nanayakkara, C. Gonzalez, M. Challacombe, P. M. W. Gill, B. Johnson, W. Chen, M. W. Wong, J. L. Andres, C. Gonzalez, M. Head-Gordon, E. S. Replogle, and J. A. Pople, GAUSSIAN 98, Revision A.6, (Gaussián, Inc., Pittsburgh, PA, 1998).

${ }^{30}$ F. Fonseca, K. Hernadi, P. Piedigrosso, J. F. Colomer, K. Mukhopadhyay, R. Doome, S. Lazarescu, L. P. Biro, P. Lambin, P. A. Thiry, D. Bernaerts, and J. B. Nagy, Appl. Phys. A: Mater. Sci. Process. 67, 11 (1998).

${ }^{31}$ L. G. Bulusheva, A. V. Okotrub, I. P. Asanov, A. Fonseca, and J. B. Nagy, J. Phys. Chem. B 105, 4853 (2001).

${ }^{32}$ M. W. Zhao, Y. Y. Xia, Y. C. Ma, M. J. Ying, X. D. Liu, and L. M. Mei, Phys. Rev. B 66, 155403 (2002). 